Takashi Sasaki • Masami Nemoto $\cdot$ Koichiro Yamasaki Naoko Tajima

\title{
Preferential transmission of maternal allele with DQA1*0301-DQB1*0302 haplotype to affected offspring in families with type 1 diabetes
}

Received: April 5, 1999 / Accepted: May 29, 1999

\begin{abstract}
To approach the possible involvement of an epigenetic mechanism in the pathogenesis of type 1 diabetes, we investigate here a parent-of-origin effect in transmission of the susceptible alleles at HLA-DQ loci by the transmission disequilibrium test. When we examined alleles of affected offspring of Japanese origin in 28 nuclear families, the maternal alleles were significantly different from the paternal alleles. Furthermore, the maternal alleles with the susceptible DQA1*0301-DQB1*0302 haplotype showed strong transmission disequilibrium with antiglutamic acid decarboxylase antibody-positive type 1 diabetes, while the paternal alleles with the same haplotype did not. This differential transmission disequilibrium of the susceptible allele was confirmed by the contingency table analysis for transmitted or nontransmitted alleles of both parental origin. The unique transmission of the susceptible allele observed supports the hypothesis that an epigenetic mechanism including genomic imprinting at the HLA-DQ region is involved in the pathogenesis and the genetic complexity of type 1 diabetes.
\end{abstract}

Key words Epigenesis · Genomic imprinting · Transmission disequilibrium test (TDT) - Parents - HLA - Insulindependent $\cdot$ Multifactorial disease

\section{Introduction}

Type 1 diabetes (also known as insulin-dependent diabetes mellitus or IDDM, OMIM\#222100) is a severe disorder characterized by complete lack of insulin secretion. Most cases with this type of diabetes develop by an autoimmune

T. Sasaki $(\square) \cdot$ M. Nemoto $\cdot$ K. Yamasaki $\cdot$ N. Tajima Department of Internal Medicine III, Jikei University School of Medicine, 3-25-8 Nishishinbashi, Minato-Ku, Tokyo 105-8461, Japan Tel. +81-3433-1111, ext. 3249; Fax +81-3-3459-1417

e-mail: stakashi@st.rim.or.jp

T. Sasaki $\cdot$ K. Yamasaki

Department of Gene Therapy, Institute of DNA Medicine, Jikei

University School of Medicine, Tokyo, Japan mechanism with disease-specific autoantibodies against components of the pancreatic islets including glutamic acid decarboxylase (GAD), but etiological heterogeneity of this type is also observed. Extensive genome-wide scan has revealed that susceptibility to type 1 diabetes is mainly determined by a HLA DQ-linked gene in combination with multiple associated genes. Epidemiological studies have revealed that incidence in different ethnic groups is globally variable (Rewers et al. 1988), but the association to the HLA-DQ region has been observed consistently in all populations examined so far.

Despite the major effect of HLA-DQ, a complex mode of inheritance has been also investigated. Observations on twins have shown that the concordant rate for development of type 1 diabetes between monozygotic twins is no more than 50\% (Tattersall and Pyke 1972; Committee on Diabetic Twins, Japan Diabetes Society 1988; Kaprio et al. 1992; Kumar et al. 1993). Furthermore, association studies have shown that excess of specific compound heterozygotes DQB $1 * 0302 / * 0201$ in Caucasian populations and DQB1 $* 0302 / * 0401$ in the Japanese was strongly associated with the disease in each population (Ikegami et al. 1992; Jenkins et al. 1992; Baisch et al. 1990; Zamani and Cassiman 1998). This complex mode of inheritance in the diseaseprone families is not explained simply by the polygenic inheritance.

Previous studies proposed that epigenetic factors interacting with the susceptibility loci could be relevant to the complex mode of inheritance in addition to environmental factors for the development of type 1 diabetes (Julier et al. 1991; Bennet et al. 1997), although some data were controversial (Wolde et al. 1993; Jos et al. 1991; Kockum et al. 1994; Deschamps et al. 1990). Heterogeneity among and within the cohort of these studies possibly causes this controversy, as the disease-specific autoantibody against GAD cannot be detected in about $30 \%$ of recent-onset type 1 diabetics. Difficulty in linkage analysis also causes this controversy because the disease associates with various alleles at the same HLA locus. Furthermore, failure in determination of exact haplotypes from identity-by-descent could also be causative for the controversy of the previous studies. 
The purpose of our present study is to determine if an epigenetic mechanism including genomic imprinting is involved in the pathogenesis of type 1 diabetes at the HLADQ region, while minimizing the foregoing difficulties that could produce controversial results. To address this question, we asked whether the parental sex would affect genetic predisposition of the susceptible alleles by conducting the transmission disequilibrium test (TDT) according to parent of origin in families with affected offspring with anti-GAD positive type 1 diabetes.

\section{Materials and methods}

Patients and families

Fifty patients with type 1 diabetes were originally recruited. Type 1 diabetes was diagnosed by the following criteria: (1) diabetic ketoacidosis/diabetic ketosis at the time of onset; (2) urinary C-peptide excretion rate less than $10 \mu \mathrm{g} / 24 \mathrm{~h}$; (3) positive for anti-GAD antibody with more than 10 units $/ \mathrm{ml}$ at any time during the course of diabetes as assayed by radioimmunoprecipitation methods (Tsuruoka et al. 1995); and (4) onset age less than 30 years old. The 50 proband with type 1 diabetes, their first-degree relatives, and 50 unrelated controls were all derived from unrelated nuclear families and of Japanese origin. The patients were from simplex families except one family with another affected offspring, but only the affected proband were analyzed in this study. Among the 50 families originally recruited, 28 families was willing to participate in this transmission study and gave their blood samples. Average onset age of the patients of these families was $19 \pm 6.9$ years (mean \pm SD; 6 30 years old), and male:female ratio was 11:17. Informed consent for the study was obtained from all the individuals in accordance with the Helsinki Declaration.

\section{Genotyping of HLA-DQB1 and DQA1 loci}

Genomic DNAs were extracted from the buffy coat of peripheral blood. Determination of HLA-DQB1 and DQA1 genotypes were performed by the PCR-SSP method as described by Olerup et al. (1993) with some modifications. Briefly, DNA was amplified by the 22 sets of sequencespecific primer for DQB1. The PCR products were then examined by $2 \%(\mathrm{w} / \mathrm{v})$ NuSieve $3: 1$ agarose gel (FMC BioProduct, Rockland, ME) or mixed agarose gel containing $0.7 \%(w / v)$ of agarose (Takara, Osaka, Japan) and $0.65 \%(\mathrm{w} / \mathrm{v})$ of Synergel (Diversified Biotech, Boston, MA). Genotypes of DQA1 were also determined using 12 sets of specific primer for DQA1 alleles. To prevent spurious results for the DQB1 genotyping, these results were also confirmed by another method with PCR-RFLP (Nomura et al. 1991). The control results obtained by the consistent and updated PCR-SSP method combined with the PCR-RFLP were confirmed by comparing to published data to ascertain whether the obtained frequency of DQB1 genotypes and DQA1-DQB1 haplotypes would agree with previously published results in the Japanese population with a large set of data.

Transmission analysis of the 28 families

DQA1-DQB1 haplotypes were determined directly based on data of each nuclear family. Frequency of haplotypes of maternal origin among affected offspring was then compared to that of paternal origin. Alleles of heterozygous parents with specific haplotypes were subsequently subject to the transmission disequilibrium test (TDT) by each parent of origin. The TDT for the susceptible haplotypes was carried out as described by Spielman et al. (1993) with the statistic $\chi_{\mathrm{td}}^{2}=(\mathrm{b}-\mathrm{c})^{2} /(\mathrm{b}+\mathrm{c})$, which was tested by the chisquare test (McNemar test). The difference in transmission frequency between paternal and maternal alleles with a specific HLA-DQ haplotype was also evaluated by the $2 \times$ 2 contingency test by calculating Fisher's exact probability when the number of data was less than five.

\section{Results}

\section{DQA1-DQB1 haplotypes of the affected offspring}

We have determined DQA1-DQB1 haplotypes of the offspring with type 1 diabetes by analyzing genotypes of both parents and offspring. This analysis was followed by identification of both transmitted and nontransmitted alleles from each parent of origin to affected offspring in each family. Table 1 summarizes haplotypes observed in the affected offspring of the 28 families according to the identityby-descent of alleles. When allelic frequency is compared according to parent of origin, the maternally transmitted haplotypes are significantly different from that of the paternally transmitted haplotypes ( 3 d.f., $\chi^{2}=15.6, P=0.0013$ ). This difference appeared to result from a significantly higher frequency of maternally transmitted DQA1*0301DQB1*0302 ( 1 d.f., $\chi^{2}=5.23, P=0.021$ ), while the other haplotypes showed no significant difference. Haplotype analysis also indicated that all the DQB $1 * 0302$ of the affected offspring were linked with DQA $1 * 0301$.

Table 1. Haplotypes of affected offspring with type 1 diabetes

\begin{tabular}{lllll}
\hline & \multicolumn{2}{l}{ Parent of origin } & \\
\cline { 2 - 4 } DQA1-DQB1 & Combined & Maternal & Paternal & $P$ \\
\hline$* 0301-* 0302$ & 18 & 13 & 5 & 0.022 \\
$* 0302-* 0401$ & 17 & 7 & 10 & \\
$* 0302-* 0303$ & 13 & 5 & 8 & \\
$* 0102-* 0604$ & 3 & 1 & 2 & \\
$* 0101-* 0501$ & 2 & 0 & 2 & \\
$* 0103-* 0601$ & 2 & 1 & 1 & \\
$* 0102-* 0604$ & 1 & 1 & 0 & \\
Total & 56 & 28 & 28 & \\
\hline
\end{tabular}

Frequency of each haplotype is indicated by both parental origin. $P$ value for the chi-square test is described only when the difference is significant 
DQB1 association of the affected offspring

To know which haplotype was actually susceptible for our Japanese patients' panel, we have compared the allele frequency of DQB1 in the affected 28 proband to unrelated 50 healthy individuals. As shown in Table 2, the allele frequency of DQB1*0302 appeared significantly higher in the type 1 diabetics than in the healthy controls. The data also showed that DQB1*0401 was positively associated with type 1 diabetes and that DQB1*0602 and DQB1*0601 were negatively associated with type 1 diabetics. In contrast, DQB1*0303 did not show any association.

Transmission analysis of susceptible alleles by parent of origin

Based on these observations, we performed further characterization on the mode of transmission of the susceptible alleles according to parental sexes. For this purpose, we

Table 2. Allelic frequencies of DQB1 in type 1 diabetes and healthy control

\begin{tabular}{|c|c|c|c|c|c|c|}
\hline \multirow{2}{*}{$\begin{array}{l}\text { DQB1 } \\
\text { allele }\end{array}$} & \multicolumn{2}{|c|}{$\begin{array}{l}\text { Type } 1 \\
\text { diabetes }\end{array}$} & \multicolumn{2}{|c|}{ controls } & \multirow[b]{2}{*}{ OR } & \multirow[b]{2}{*}{$P$} \\
\hline & $n$ & $(\%)$ & $n$ & $(\%)$ & & \\
\hline$* 0302$ & 18 & 32.1 & 17 & 17.0 & 2.31 & 0.029 \\
\hline *0401 & 17 & 30.4 & 11 & 11.0 & 3.52 & 0.003 \\
\hline *0303 & 13 & 23.2 & 15 & 15.0 & & \\
\hline *0604 & 4 & 7.1 & 6 & 6.0 & & \\
\hline$* 0601$ & 2 & 3.6 & 24 & 24.0 & & \\
\hline$* 0501$ & 2 & 3.6 & 4 & 4.0 & & \\
\hline *0602 & 0 & 0.0 & 5 & 5.0 & & \\
\hline *0201 & 0 & 0.0 & 0 & 0.0 & & \\
\hline Others & 0 & 0.0 & 18 & 18.0 & & \\
\hline Total & 56 & 100.0 & 100 & 100.0 & & \\
\hline
\end{tabular}

$n$, number of allele. Odds ratio (OR) and the $P$-value are described only when positive association is significant. OR for DQB1*0303 is 1.71 without significant difference; $P=0.199$ tested if the DQA1*0301-DQB1*0302 allele of one parental sex of origin would transmit differentially to the diabetic offspring when compared to the other parental sex of origin by the TDT. Table 3 shows the transmission frequency of DQA1*0301-DQB1*0302 from both parents. Fifteen mothers and 10 fathers had the allele with DQA1 *0301-DQB1*0302 haplotype in these 28 unrelated families. It appeared that all the 15 mothers and the 8 fathers of these were heterozygous with this susceptible haplotype. By examining these 15 heterozygous mothers with the TDT, 13 maternal alleles actually transmitted to the diabetic offspring but only 2 alleles did not. Therefore, the TDT reveals that the maternal DQA1*0301-DQB1*0302 showed significant transmission disequilibrium, or a higher chance of transmission to the diabetic offspring than the expected equal chance for transmission and nontransmission $\left(\chi_{\mathrm{td}}^{2}=\right.$ $8.06, P=0.0045)$. Examination of the 8 fathers heterozygous for DQA1*0301-DQB1*0302, on the other hand, indicated that 3 alleles transmitted to the affected offspring while 5 alleles did not. This result indicates that the paternal DQA1*0301-DQB1*0302 had no significant difference in transmission frequency to the affected offspring compared to the Hardy-Weinberg random transmission.

The significant difference in transmission frequency between the paternal and the maternal alleles with the DQA1*0301-DQB1*0302 haplotype was also investigated when the transmission table was analyzed by the $2 \times 2$ contingency analysis (Table 3; odds ratio, 10.8; Fisher's exact probability, 0.025). The TDT for the DQA1*0301DQB1*0302 alleles showed increased frequency of transmission to the affected offspring when maternal and paternal transmission were combined, but the statistics indicated no significance $(P=0.061)$.

TDT analysis of the DQA1*0302-DQB1*0401 allele indicated that alleles with this haplotype showed significant transmission disequilibrium with type 1 diabetes. However, no difference was observed in transmission frequency of the alleles between both parents of origin (Table 4).

Table 3. Transmission of alleles with DQA $1 * 0301-\mathrm{DQB} 1 * 0302$

\begin{tabular}{lllllll}
\hline Parent of origin & Transmitted & Nontransmitted & Homozygotes & Total & $\chi_{\text {td }}^{2}$ \\
\hline Mother & 13 & 2 & 0 & 15 & 8.643 \\
Father & 3 & 5 & 2 & 10 & 0.003 \\
Combined & 16 & 7 & 2 & 25 & N.A. & 3.522 \\
\hline
\end{tabular}

N.A., not applicable

The maternal allele with DQA1*0301-DQB1*0302 haplotype shows significant transmission disequilibrium $\left(\chi_{\text {td }}^{2}=8.64 ; P=0.003\right)$, but the paternal allele does not. When the both parental alleles are analyzed by $2 \times 2$ contingency according to their transmission and parent of origin, significant difference is observed (Fisher's exact probability is 0.025 )

Table 4. Transmission of alleles with the DQA $1 * 0302-\mathrm{DQB} 1 * 0401$

\begin{tabular}{llllrr}
\hline Parent of origin & Transmitted & Nontransmitted & Homozygotes & Total & $\chi_{\text {td }}^{2}$ \\
\hline Mother & 5 & 0 & 2 & 7 & 3.682 \\
Father & 8 & 2 & 1 & 11 & 0.055 \\
Combined & 13 & 2 & 3 & 18 & 3.600 \\
\hline
\end{tabular}

The alleles with the DQA1*0302-DQB1*0401 haplotype showed no difference between parent of origin, although the transmission disequilibrium is significant $\left(\chi_{\mathrm{td}}^{2}=8.64 ; P=0.003\right)$ 


\section{Discussion}

This study aimed to investigate the unique transmission of the susceptible marker allele that is identified by the HLADQA1-DQB1 haplotype. A large number of individuals is needed to accomplish the association study with the highly heterozygous DQB1 locus, but the sample size was not sufficient for such an association analysis in this study. Basically, the present study did not focus on the populationassociation study that specifies the HLA-disease association. Therefore, we have excluded some populationassociation data and focused only on the family-based data. However, the alleles with DQB $1 * 0302$ and DQB $1 * 0401$ in the panel of this study showed association with type 1 diabetes as well as that observed previously with a large number of patients (Ikegami et al. 1992; Yasunaga et al. 1996; Kawasaki et al. 1998). The allele with DQB1*0302, specifically the DQA1*0301-DQB1*0302 haplotype, has been extensively investigated and shown to associate with type 1 diabetes in various populations. Furthermore, this common haplotype corresponds to the alleles with the specific amino acid variation at position 57 in the DQB1 gene whose frequency correlates with the incidence of type 1 diabetes in many populations (Dorman et al. 1990).

When we examined the frequency of the DQA1*0301DQB1*0302 in the affected offspring, the maternally transmitted alleles were observed more frequently than the paternally transmitted alleles. Therefore, it is critical for evaluation of the epigenetic hypothesis of type 1 diabetes to ask if the susceptible allele common to all ethnicity would differentially transmit to affected offspring according to parent of origin in the 28 families. By the TDT analysis in this study, we have found that the maternal alleles with DQA1*0301-DQB1*0302 haplotype would preferentially transmit to the affected offspring while the paternal DQA1*0301-DQB1*0302 alleles would not. When data of both parents of origin were combined, the alleles with DQA1*0301-DQB1*0302 even showed an increased chance of transmission to the affected offspring, but this result was not significant $(P=0.061)$. Because the paternal allele shows no increase in frequency of transmission to the affected offspring, the transmission disequilibrium of combined alleles might be weak, and relatively large numbers of families are required for statistically significant detection of this observation.

When the transmission was analyzed according to the parent of origin, the maternal alleles with the DQA $1 * 0301$ DQB1*0302 haplotype showed strong transmission disequilibrium with type 1 diabetes, while the paternal alleles with the same haplotype did not. No such study according to the parent of origin has been investigated using the TDT so far. As the TDT considers transmission of the marker allele only when the parents are heterozygous in each family, the results are not influenced by sampling error, including population stratification. This differential transmission between maternal and paternal DQA1*0301-DQB1*0302 alleles was also investigated when Fisher's exact probability analysis was performed with the $2 \times 2$ contingency table.
Therefore, it is unlikely that our observation has resulted from sampling error and that the TDT should be even more powerful to detect the transmission disequilibrium of alleles with specific parent of origin according to their sex when combined with the $2 \times 2$ contingency analysis.

From these observations, we hypothesize that an epigenetic mechanism including genomic imprinting at the HLADQ linked gene might be involved in the pathogenesis of type 1 diabetes. In this hypothesis, the maternally transmitted allele with the DQA1*0301-DQB1*0302 haplotype might be inactive in their offspring. The inactive alleles that were maternally transmitted and imprinted therefore could not compensate sufficiently with the paternally transmitted allele with the DQA1*0302-DQB1*0401 haplotype, which might be invariably inactive despite the parent of origin. Maternal-fetal HLA compatibility can also be related to the development of type 1 diabetes and is an alternative explanation, as implicated in other genetic complex diseases (Gill 1993; Nelson et al. 1993).

In spite of suggestive observations and the theoretical possibility for the epigenetic mechanism (MargaritteJeannin et al. 1995; Bonneuli et al. 1997), some previous studies even with a large data set have failed to detect the parent-of-origin effect in the transmission of specific HLADQ, DR alleles (Undlien et al. 1995) in families with type 1 diabetes. This controversy can be the result of the potential heterogeneity of type 1 diabetes in terms of etiology. It may also account for misrelated haplotypes caused by estimation from the linkage disequilibrium frequently observed in the general population. In the present study, to the contrast, we examined the families with type 1 diabetics who had antiGAD autoantibody, and determined directly the actual HLA-DQ haplotypes to avoid these problems.

The TDT assumes that the segregation distortion during meiosis should not occur at the examined locus. If the segregation distortion is present at the HLA-DQ region and causes the disparity in the transmission frequency between paternal and maternal alleles, it must also occur in diseaseunrelated families and unaffected sibs. In this study, the parental alleles with DQA1*0301-DQB1*0302 showed no significant transmission distortion when we observed the transmission to the unaffected sibs (data not shown). To confirm the involvement of the preferential transmission related to parental sex of origin, however, we need to perform further tests to exclude transmission distortion phenomenon at the HLA-DQ region by examining families without diabetes.

Transmission disequilibrium by itself is not able to demonstrate whether the detected difference of transmission between both parental alleles is related to the pathogenesis of type 1 diabetes. However, studies on cases with paternal isodisomy of chromosome 6 have demonstrated strong evidence that an imprinted gene linked to the HLA region is implicated in a mechanism of diabetes mellitus and an autoimmune disorder (Abramowicz et al. 1994; Whiteford et al. 1997; Arthur et al. 1997; Temple et al. 1995). This finding indicates that deregulation of genomic imprinting at the HLA-DQ region should have profound effects on pancreatic beta cell function and the immune system. This result 
also strongly supports our hypothesis that genomic imprinting at the HLA-DQ region may be involved in the genetic mechanism of pancreatic islet cell dysfunction with the pathogenesis of type 1 diabetes.

Acknowledgments We express special thanks to Professor Stanley M. Gartler at the University of Washington in Seattle and Professor Yoshikatsu Eto at Jikei University for their important suggestions. T.S. was supported by a grant-in-aid for scientific research from the Ministry of Education, Science, Sports and Culture of Japan.

\section{References}

Abramowicz MJ, Andrien M, Dupont E, Dorchy H, Parma J, Duprez L, Ledley FD, Courtens W, Vamos E (1994) Isodisomy of chromosome 6 in a newborn with methylmalonic acidemia and agenesis of pancreatic beta cells causing diabetes mellitus. J Clin Invest 94: 418421

Arthur EI, Zlotogora J, Lerer I, Dagan J, Marks K, Abeliovich D (1997) Transient neonatal diabetes mellitus in a child with invdup(6)(q22q23) of paternal origin. Eur J Hum Genet 5: 417-419

Baisch JM, Weeks T, Giles R, Hoover M, Stastny P, Capra JD (1990) Analysis of HLA-DQ genotypes and susceptibility in insulindenpendent diabetes mellitis. N Engl J Med 322: 1836-1841

Bennet ST,Wilson AJ, Esposito L, Bouzekri N, Undlien DE, et al. (1997) Insulin VNTR allele-specific effect in type 1 diabetes depends on identity of untransmitted paternal allele. Nat Genet 17: 350-352

Bonneuli N, Clerget A, Clerget-Darpoux F (1997) Variable age at onset in insulin-dependent diabetes mellitus, by the marker-association-segregation- $\chi^{2}$ method. Am J Hum Genet 61: 223-227

Committee on Diabetic Twins, Japan Diabetes Society (1988) Diabetes mellitus in twins: a cooperative study in Japan. Diabetes Res Clin Pract 14: 4271-4280

Deschamps I, Hors J, Clerget-Darpoux F, Gardais E, Robert JJ, Marcelli-Berge A, Lestradet H, Dausset J (1990) Excess of maternal HLA-DR3 antigens in HLA DR3,4 positive type 1 (insulin-dependent) diabetic patients. Diabetologia 33: 425-430

Dorman JS, LaPorte RE, Stone RA, Trucco M (1990) Worldwide differences in the incidence of type I diabetes are associated with amino acid variation at position Proc Natl Acad Sci USA 87: 73707374

Gill TJ (1993) Maternal-fetal interactions and disease. N Engl J Med 329: 500-501

Ikegami H, Kawaguchi Y, Yamato E, Kuwata S, Tokunaga K, Noma Y, Shima K, Ogihara T (1992) Analysis by the polymerase chain reaction of histocompatibility leukocyte antigen-DR9-linked susceptibility to insulin-dependent diabetes mellitus. J Clin Endocrinol Metab 75: 1381-1385

Jenkins D, Penny MA, Uchigata Y, Jacobs KH, Mijovic CH, Hirata Y, Otani T, Fletcher J, Bradwell AR, Barnett AH (1992) Investigation of the mode of inheritance of insulin-dependent diabetes mellitus in Japanese subjects. Am J Hum Genet 50: 1018-1021

Jos J, Farkas D, De Tand M-F, Cartron J, Cohen-Hagenhauser O, Tozzo E, Deschamps I (1991) DNA polymorphism analysis of HLA class II genes in unrelated children and in first-degree relatives with type I diabetes. Diabetes Res 18: 53-59

Julier C, Hyer RN, Merlin F, Soularue P, Briant L, Cathelineau G, Deschamps I, Rotter JI, Froguel P, Boitard C, Bell JI, Lathrop GM (1991) Insulin-IGF2 region on chromosome 11p encodes a gene implicated in HLA-DR4-dependent diabetes susceptibility. Nature
354: $155-159$

Kaprio J, Toumilehto J, Koskenvuo M, Romanov K, Reumanen A, Eriksson J, Stenård J, Kesäniemi YA (1992) Concordance for type 1 (insulin-dependent) and type 2 (non-insulin dependent) diabetes mellitus in a population-based cohort of twins in Finland. Diabetologia 35: 1060-1067

Kawasaki E, Noble J, Erlich H, Mulgrew CL, Fain PR, Eizenbarth GS (1998) Transmission of DQ haplotypes to patients with type 1 diabetes. Diabetes 47: 1971-1973

Kockum I, Wassmuth R, Holmberg E, Michelsen B, Lernmark A (1994) Inheritance of MHC class II genes in IDDM studied in population-based affected and control families. Diabetologia 37: 11051112

Kumar D, Gemayel NS, Deapen D, Kapadia D, Yamashita PH, Lee M, Dwyer JH, Roy-Burman P, Bray GA, Mack TM (1993) North American twins with IDDM. Genetic, etiological, and clinical siginificance of desease concordance according to age, zygosty, amd interval after diagnosis in first twin. Diabetes 42: 1351-1363

Margaritte-Jeannin P, Clerget-Darpoux F, Hors J, Deschamps I (1995) Testing parental imprinting in insulin-dependent diabetes mellitus by the marker-association-segregation-chi ${ }^{2}$ method. Am J Hum Genet 56: 1080-1087

Nelson JL, Hughes KA, Smith AG, Nisperos GG, Branchaud AM, Hansen JA (1993) Maternal-fetal disparity in HLA class II alloantigens and the pregnancy-induced amelioration of rheumatoid arthritis. N Engl J Med 329: 466-471

Nomura M, Ota M, Tsuji K, Inoko H (1991) HLA-DQB1 genotyping by a modified PCR-RFLP method combined with group-specific primers. Tissue Antigens 38: 53-59

Olerup O, Aldener A, Fogdell A (1993) HLA-DQB1 and -DQA1 typing by PCR amplification with sequence-specific primers (PCRSSP) in 2 hours. Tissue Antigens 41: 119-134

Rewers M, LaPorte RE, King H, Tuomilehto J (1988) Trends in the prevalence and incidence of diabetes: insulin-dependent diabetes mellitus in childhood. World Health Stat Q 41: 178-189

Spielman RS , McGinnis RE, Ewens WJ (1993) Transmission test for linkage disequilibrium: the insulin gene region and insulin-dependent diabetes mellitus (IDDM). Am J Hum Genet 52: 506-516

Tattersall RB, Pyke DA (1972) Diabetes in identical twins. Lancet 2: 1120-1125

Temple IK, James RS, Howell WM, Baum DJ, Shield JPH (1995) An imprinted gene(s) for diabetes? Nat Genet 9: 110-112

Tsuruoka A, Matsuba I, Toyota T, Isshiki G, Nagataki S, Ikeda Y (1995) Antibodies to GAD in Japanese diabetic patients: a multicenter study. Diabetes Res Clin Pract 28: 191-199

Undlien DE, Akselsen HE, Joner G, Dahl-Jorgensen K, Aagenaes O, Sovic O, Thorsby E, Ronningen KS (1995) No difference in the parental origin of susceptibility HLA class II haplotypes among Norwegian patients with insulin-dependent diabetes mellitus. Am J Hum Genet 57: 1511-1514

Whiteford ML, Narendra A, White MP, Cooke A, Wilkinson AG, Robertson KJ, Tolmie JL (1997) Paternal uniparental disomy for chromosome 6 causes transient neonatal diabetes. J Med Genet 34: $167-168$

Wolde ST, Breedveld FC, De Vries RRP, D'amaro J, Rubenstein P, Schreuder GMT, Claas FHJ, Van Rood JJ (1993) Influence of noninherited maternal HLA antigen on occurrence of inherited arthritis. Lancet 34: 200-202

Yasunaga S, Kimura A, Hamaguchi K, Ronningen KS, Sasazuki T (1996) Different contribution of HLA-DR and -DQ genes in susceptibility and resistance to insulin-dependent diabetes mellitus (IDDM). Tissue Antigens 47: 37-48

Zamani M, Cassiman J-J (1998) Reevaluation of the importance of polymorphic HLA class II alleles and amino acids in the susceptibility of individuals of different populations to type I diabetes. Am J Med Genet 76: 183-194 